\title{
Preparation of a hybrid photopolymer for stereolithography
}

\author{
Yining Zhao ${ }^{1,2 a}$, Mingshan Yang ${ }^{1,2, b^{*}}$, Ran Ding ${ }^{2, \mathrm{c}}$, Qisong Shi ${ }^{2, \mathrm{~d}}$, Ming Zhao ${ }^{2, \mathrm{e}}$ \\ ${ }^{1}$ School of Material Science and Engineering, Beijing University of Chemical Technology, Beijing \\ 100029, China \\ ${ }^{2}$ School of Material Science and Engineering, Beijing Key Lab of Special Elastomer Composites \\ Materials, Beijing Institute of Petrochemical Technology, Beijing 102617, China

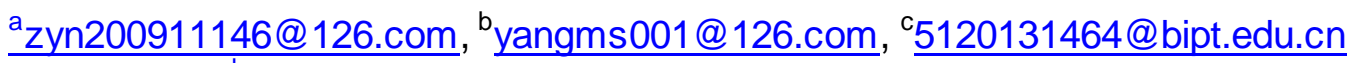 \\ d shiqisong@bipt.edu.cn, ㄹ.Zhaoming888@bipt.edu.cn
}

Keywords: free radical-cationic hybrid; interpenetrating network, formulation optimized.

Abstract. New free radical-cationic hybrid photopolymer, consisting of acrylate-based and epoxy-based photosensitive resin for stereolithography was prepared. The influence of the initiator sort and amount on the curing degree of the photopolymer was investigated. Photopolymerization process, degradation temperature, dynamic mechanical thermal properties of the Interpenetrating polymer networks (IPNs) formed are characterized by using FTIR spectroscopy, TG and DMA. The results indicate curing reaction is reacting sufficiently and all performance is better when the ratio of acrylate / epoxy resin is 1:0.75. And post-curing could also improve properties of hybrid resin after UV curing.

\section{Introduction}

Referred to as solid freeform manufacturing, Rapid Prototyping has become increasingly important to designers and manufacturers in the past two decades. One of the most commonly used methods of rapid manufacturing technology is stereolithography, in which intricate parts of a plastic monomer are directly built by photopolymerization ${ }^{[1]}$. The resin composition used consists of: photoinitiators, prepolymer reactants and additives. The photosensitive resin systems can be classified into three categories: free radical system, cationic and hybrid systems. Free radical systems based on acrylate and methacrylate monomers have relative insensitivity to temperature and humidity changes, high photosensitivity and a high speed of polymerization, but poor dimensional stability. The most commonly studied in the cationic systems of interest are epoxies and vinyl ethers. Cationic exhibits high active center concentrations. However, anhydrous conditions also limit the development of cationic systems. Hybrid systems have also been proposed, comprising both acrylate compounds and epoxy of vinyl ether compounds forming an interpenetrating network. Hybrid system has a synergistic effect, showing the advantages both of radical system and cationic systems, avoiding their disadvantages. This paper aims to discuss about the photopolymerization behavior and the compatibility of the light-curing efficiency of the hybrid system ${ }^{[2-5]}$.

\section{Experimental Procedure}

\section{Materials}

3,4-Epoxycyclohexyl-methyl3'4'-cyclohexylcarboxylate(CY179) was purchased from the Huntsman. E-44 epoxy resin was supplied by Yueyang Baling Petrochemical Corporation. Ethoxylated Trimethylolpropane Triacylate.(SR454) were provided by Sartomer. PAG201 (mixed triarylsulfonium hexafluoroantimonate salts) and PAG202 (mixed triarylsulfonium hexafluorophosphate salts) was gratefully received as gifts from the Changzhou Tronly New Electronic Materials Co.Ltd. A sample of Irgacure 184 was kindly supplied by Tianjin Jiuri Chemical Co., Ltd. 


\section{Formulation preparation}

Photocurable formulations were premixed by mixing epoxy resin and acrylic resin in different ratios in a beaker under ambient condition by stirring for $30 \mathrm{~min}$ with a magnetic stirrer at. $25^{\circ} \mathrm{C}$. The appropriate amount of Initiator was added, and then the mixture was sonicated for 30min to dissolve initiator. Then the mixture was again stirred for $20 \mathrm{~min}$ at room temperature to further mix.

Test

The kinetic of the photopolymerization was determined by Fourier-transform IR (FTIR) spectroscopy in the range of 400-4000 $\mathrm{cm}^{-1}$, using a Thermo-Nicolet 6700 instrument. The acrylic's double-bond conversion was followed by monitoring the $\mathrm{C}=\mathrm{C}$ double-bond peak, centered at $1635 \mathrm{~cm}^{-1}$, while the epoxy group's conversion was followed by -monitoring the decrease in the absorbance in the $910 \mathrm{~cm}^{-1}$ region.

A UV-cured sample was accurately weighted, and added to the Soxhlet extractor with acetone as extraction agent for $12 \mathrm{~h}$, then dried in the oven until its weight was constant $\left(\mathrm{m}_{2}\right)$. Gel content of the UV-cured sample was calculated by Eq (1)

$$
\text { Gel content }=\frac{\mathrm{m}_{2}}{\mathrm{~m}_{1}} \times 100 \%
$$

The tangent of the phase angle of the samples were characterized as a function of temperature by dynamic mechanical analysis (DMA) using a instrument Anton Paar -MCR301-Physica rheometer polymer dynamic mechanical properties in tensile mode, The samples were subjected to an frequency of $1 \mathrm{~Hz}$ in the temperature range of $25 \sim 180{ }^{\circ} \mathrm{C}$ at a heating rate of $5{ }^{\circ} \mathrm{C} / \mathrm{min}$.

Weight loss of the resin samples in the pyrolysis process was performed with TGA/DSC1 thermal gravimetric analysis instrument ( Mettler Toledo) under the nitrogen atmosphere at a heating rate of $20^{\circ} \mathrm{C} \mathrm{min}-1$ in the temperature range of $25-700^{\circ} \mathrm{C}$.

Dumbbell-shaped test sample were used for tensile testing $(75 \mathrm{~mm} \times 3.2 \mathrm{~mm} \times 2 \mathrm{~mm}$, ASTM:D638), the extension speed is $1 \mathrm{~mm} / \mathrm{min}$. Rectangle bars were carried out for flexural testing $(80 \mathrm{~mm} \times 10 \mathrm{~mm} \times 4 \mathrm{~mm}$, ASTM:D790) and charpy impact testing (80mm×10 mm×4mm, GB/T1043-2008).

\section{Results and discussion}

The influence of initiator category on the performance of UV curing In order to investigate the influence of initiator on the performance of UV curing, we selected two kinds of initiator, PAG201 (mixed sulfonium hexafluoroantimonate salt) and PAG202 (mixed sulfonium hexafluorophosphate salts) which contain different central ion, to observe the initiating effect of initiator on the double bond of radical component and epoxy bond of cationic component in the photosensitive resin. The result is shown in Figure. 1.
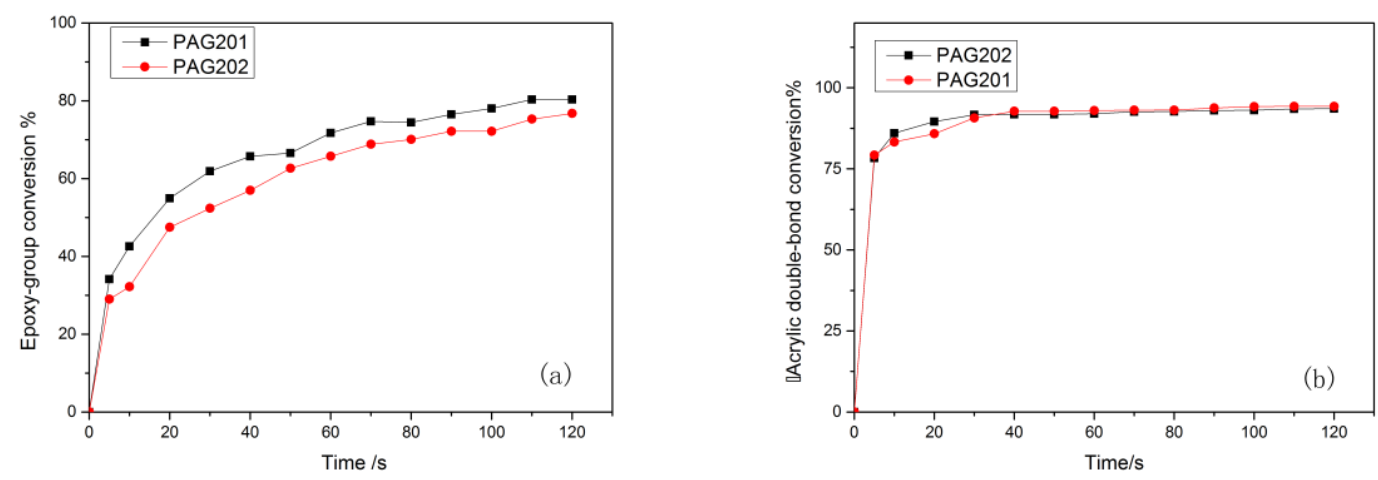

Fig. 1 (a)The influence of initiator category on the epoxy-group conversion during UV curing (b)The influence of initiator category on the acrylic double-bound conversion during UV curing 
The absorption capability of Triarylsulfonium hexafluorophosphate salt is remarkable at $365 \mathrm{~nm}$. Subjected to UV radiation, it will produce free radical and cation, which would induce free radical and cationic polymerization of acrylate of photosensitive resin and epoxy, while the 184 complex also make this initiating system have a higher initiating efficiency. Shown in Figure 1, the initiating effects of cationic initiator PAG021 on Epoxy bond and Double bond are better than that of PAG202 since the nucleophilicity of pairing anion $\mathrm{SbF}^{-6}$ of triarylsulfonium salt is weaker than that of $\mathrm{PF}^{-6}$. However, the photosensitive resin polymerized by the PAG201 is presenting severe yellowing after UV curing and rapid prototyping machines laser curing. So the subsequent experiments will use PAG202.

The influence of the amount of photoinitiator on the curing degree of the cured sample

The gel content served as an index to inspect the effect of the amount of polymerization effect. Taking advantage of triarylsulfonium hexafluorophosphate as photoinitiator and keep a constant ratio of matrix resin, the curing rate of the resin system is relevant to amount of the initiator.

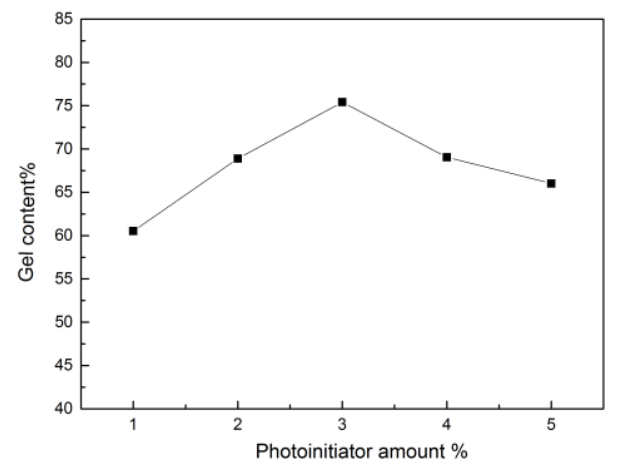

Fig. 2 The effect of the amount of photoinitiator on the gel content.

It was observed from Fig. 2 that the gel content similarly linearly increased with the increase of the amount of initiator at the beginning of the UV-curing stage, and reaches the highest at 3\% .It is due to low concentration of proton acid produced by photodecomposition when the concentration is less than $3 \%$. However, when the initiator concentration is above $3 \%$, the polymerization rate slows down instead. Because according to the Lambert-Beer's Law, the intensity of permeation light decreased in an exponential law. The number of photons absorbed by the surface of the resin initiator increases and photosensitization at the bottom is not sufficient. Additionally, the cross-linked network has formed will limit the initiator activity and the movement of molecular segments when the resin system reaches to a certain extent. So that $3 \%$ concentration is an appropriate concentration.

Dynamic mechanical thermal properties of hybrid photopolymer

Dynamic mechanical thermal properties are further obtained to evaluate the relationship of the relationship of the cured sample's structure and property. The acrylic/epoxy monomer ratio was varied, ranging from 1:0.5 to $1: 1.5(\mathrm{wt} / \mathrm{wt}-\%)$ in the photocurable formulation.DMA measure the deformation of a material in response to oscillating forces. As shown in Fig. 4, As the proportion of epoxy resin increasing, two Tg could be observed, while the range of transition temperature broadening. Meanwhile, $\tan \delta$ peaks height increase along with the proportion of epoxy increase which indicates that the crosslink density of the material reduce. This phenomenon is due to the more enhanced local activity of the molecule. The IPN is prepared at the ratio of acrylate/epoxy is 1:0.5 and 1:0.75 with a broad $\mathrm{Tg}$ peak could be observed, indicating complicated polymer-network interpenetrating formed. And the narrower peak width shows that the components of the product are not only fully compatible but also should have a strong interaction during the curing process. We may Infer that random copolymer might synthesized and played a role as a compatibilizer, a more uniform network formed. 


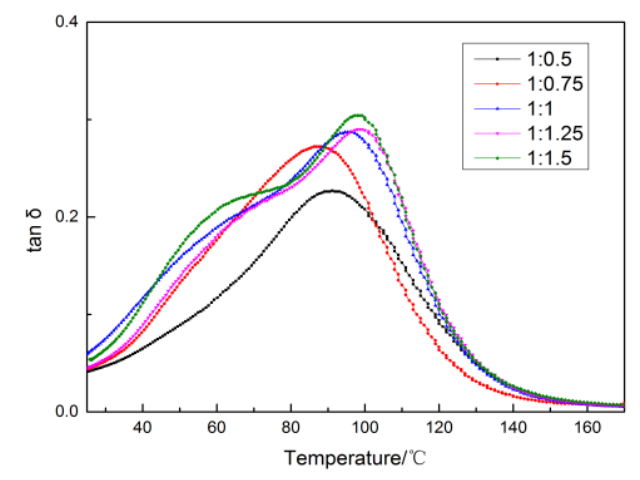

Fig .3 Effect of ratio of acrylate/epoxy on the dynamic mechanical thermal properties

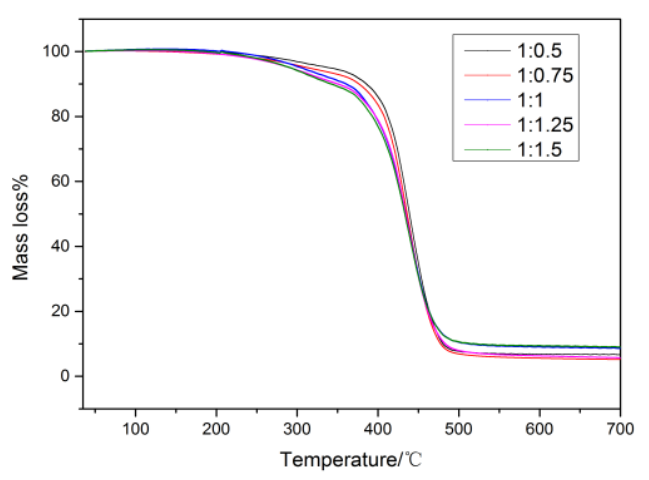

Fig .4 TG curves of different ratio of acrylate/epoxy cured films.

Thermogravimetric analysis of hybrid photopolymer

Thermal gravimetric analysis (TG) is commonly used to evaluate the thermal stability of different materials. It could characterize decomposition of polymers and structural materials at different temperatures. TGA results of different formulation are shown in Fig.3. The radial-cationic hybrid system cured samples have shown a small weight loss platform at $240{ }^{\circ} \mathrm{C}$ to $360{ }^{\circ} \mathrm{C}$, mostly involved the decomposition of unreacted initiator, prepolymer and reactive diluents. Then the weight of all the samples decreased dramatically in the range of 390 $490^{\circ} \mathrm{C}$, and a lower char yield is achieved.

\section{Conclusions}

In the system of acrylate/epoxy hybrid system, the cationic initiator PAG202 has high initiating activity without yellow products. The cured resin gel fraction initiated by PAG202 presents a trend that increasing first and then decreasing, and reaches the highest at 3\% By studying thermal stability and dynamic mechanical properties of acrylate /epoxy resin with five different ratios, we believe that the performance of curing is better when the ratio of acrylate / epoxy resin is 1:0.75.

\section{Acknowledgements}

This research was financially supported by the Graduate Innovation Foundation (15033981002/050), the University Student Research Training (URT) Project08032082005/021, Project of Outstanding Professors and Management Experts(BIPT-POPME-2014) of Beijing Institute of Petrochemical Technology, and by Scientific Research Project of Beijing Municipal Commission of Education(KM201510017004).

\section{References}

[1] P.J. Bartolo, stereolithography.(2011) 113-140.

Reference to a book:

[2] B.Steinmann, Praroman,U.S.Patent 6989,225 B2(2006)

[3] A.G.a.A.M.C. Esposito Corcione*, Photopolymerization kinetics of an epoxy based resin for stereolithography, (2003).

[4] J.R. Nowers, B. Narasimhan, The effect of interpenetrating polymer network formation on polymerization kinetics in an epoxy - acrylate system, Polymer 47 (2006) 1108-1118. 
[5] M. Sangermano, G. Malucelli, G. Delleani, A. Priola, Bicyclo-orthoester as a low-shrinkage additive in cationic UV curing, Polymer International 56 (2007) 1224-1229. 